\title{
Geoinformation modeling of near-Earth space: current tasks and future prospects
}

\author{
P.Yu. Orlov ${ }^{*}$, M.A. Boyarchuk and I.G. Zhurkin \\ Moscow State University of Geodesy and Cartography, Moscow, Russia
}

\begin{abstract}
The history of space exploration and current near-Earth space objects population are described in this paper. Moreover, the necessity of GIS technologies application to solve space industry tasks and the development of corresponding GIS are explained. The legal and regulatory issues of the near-Earth space GIS project development are touched upon, as well as the classification and options for the target purpose of the system. The stages of technological search and development are presented, as a result of which the Cesium library was chosen. Based on the results of discussions, a decision is made to expand the limits of the modeling space to the outer boundaries of the Hill sphere. In addition, it is noted that the objects of geoinformation modeling of near-Earth space should be not only space objects, considered as three-dimensional, but also the physical fields of the Earth. The results of performance evaluation experiments on SGP4/SDP4 based software tool for predicting space objects position are shown, and the accuracy of this model itself is assessed by reference GPS coordinates. Possible ways of industry tasks that could be solved using the developed near-Earth space GIS are presented; promising routes of the future development including DISCOS data are indicated.
\end{abstract}

\section{Introduction}

To begin with, it is important to define the specific terms, definitions and acronyms that will be referred to this paper $[1,2]$.

Near-Earth space (NES) - the space limited by a sphere which radius is equal to the average distance from the Earth to the Moon $(\sim 384,000 \mathrm{~km})$. Moreover, its lower boundary under international law is determined by the height of $100 \mathrm{~km}$. However, there is opinion that the near-Earth space upper border should be set to $51,000 \mathrm{~km}$, and the distance from it up the Moon's average orbit should be referred to a sublunar space [2]. In addition, the statement about the outer border of terrestrial gravity zone at an altitude, depending on the author, from 930 to $1,500 \mathrm{~km}$ looks doubtful: at these altitudes, an exosphere is observed the external component of the Earth's atmosphere, extending to $\sim 190,000 \mathrm{~km}$, and from an altitude of $\sim 1,500 \mathrm{~km}$ (in average) and up to $\sim 15,000 \mathrm{~km}$, the Earth's (Van Allen) radiation belt is located, in its shape resembling a two-layer toroid [3].

Space object (SO) - a celestial body of natural or artificial origin which is located in the outer space (including NES). The natural SO are: planets, asteroids, comets, meteorites and

* Corresponding author: knightrider3e0@gmail.com 
other things descending from them. The artificial space objects include spacecrafts, their parts, as well as launch vehicle stages and other man-made fragments that are a part of space debris.

Space debris (SD) - all artificial objects in near-Earth space that do not operate for the intended purpose. These objects are hazardous for both spacecrafts and the Earth [4].

Space exploration which began more than 60 years ago may be divided into three stages $[5,6]$ :

- First stage (1957 - 1975): Stage of political ambitions. During this period the mankind managed to go outside its native planet and see the endless expanse of the Universe. The result of the Space Race between the Soviet Union and the United States were: the First artificial Earth satellite, the First manned spaceflight, the First extravehicular activity and the First landing on the Moon. However, these achievements were used to enhance the prestige of the countries participating the race rather than practical use.

- Second stage (1975 - 1995): Stage of practical problems. The mankind begins to explore the space consciously and use for the needs of humanity. The constellations of global navigation systems, like GPS and GLONASS, are formed. Regular studies of the Earth's surface and its atmosphere with remote sensing techniques begin. A discipline of space geodesy is developing at that moment. The first satellite measurements of the geopotential are being made. The number of artificial space objects in orbit reaches several thousand. In 1978, NASA employee Donald Kessler, based on his predictions, put forward a hypothesis suggesting the onset of a cascade effect after the first collisions of space objects between the 1980s and 2000 s, resulting in an increase in the number of space debris fragments [7]. In 1987, the European Space Agency approved the Space Debris Working Group, making the framework for the future DISCOS project $[8,9]$. Nevertheless, the issues of space ecology are left aside.

- Third stage (1995 - the present): Stage of uncertainty. The absence of clear public policy and precise plan to explore outer space in the majority of space-faring nations leads to greater technogenic pollution of near-Earth space. The number of catalogued space objects exceeds 15,000 units in late 2015, 18,000 units in late 2018 and reaches 21,901 in early 2021 [10], due to the uncontrolled growth and lack of a comprehensive and regular monitoring. In addition the amount of SO that can't be observed from the Earth (less than $10 \mathrm{~cm}$ ) according to some estimations is above 200,000 [11]. Collisions of spacecrafts with space debris in orbit happen more often than even before which in turn result in an increase of near-Earth space pollution. The first accident of such type was the collision of Russian communication satellite Cosmos-2251 (which was already inactive at that time) and Iridium-33 designed for satellite communications $[12,13]$. Chinese and Indian antisatellite missile tests in 2007 and 2019, respectively, also produced more than 3,000 debris fragments in total $[14,15]$.

According to the DISCOSweb information and analytical system of the European Space Agency [16], as of October 14th, 2020, the following situation was observed in near-Earth space:

- Number of satellites still in orbit: about 5,900.

- Number of active satellites: about 3,200.

- Number of tracked space debris fragments: about 27,850.

- Estimated number of destructions, explosions, collisions or anomalous events leading to fragmentation: more than 550 .

- Total mass of all space objects in Earth orbit: more than 9,000 tons. 
- Number of space debris in orbit, estimated with statistical models: 34,000 objects over $10 \mathrm{~cm}$ in diameter; 900,000 objects from 1 to $10 \mathrm{~cm}$ in diameter; $128,000,000$ objects from $1 \mathrm{~mm}$ to $1 \mathrm{~cm}$ across.

Currently, the commercialization of the space industry leads to the near-Earth space pollution with various objects of rocket and space technology, for example, small satellites of the CubeSat class (picosatellites), the number of which has already exceeded one thousand in orbit. In the next 10 years, state and private space agencies are planning to launch from 2,000 to 10,000 picosatellites into the most loaded LEO region. These satellites have no thrusters, which mean that their orbital parameters cannot be changed in the event of an emergency [17]. This is exactly how the collision of the NEE-01 "Pegaso" spacecraft belonging to the Ecuadorian Civilian Space Agency with the upper stage of the Tsyklon-3 launch vehicle happened. The satellite was not destroyed, but was disoriented and unable to communicate with the ground control station. Only 8 months later on January 25th, 2014 a communication line was restored, using its second spacecraft NEE-02 "Krysaor" as an orbital repeater. Search and identification of such satellites is also challenging. In addition, there is a constant increase in both the volume of data from ground-based surveillance stations, and data from remote satellite measurements of objects on Earth's surface and in space around it. Geoinformatics is currently an integrator of many disciplines and technologies that handle spatially coordinated data. The interaction of geoinformatics, ballistics, celestial mechanics, space navigation, astrometry and telecommunications reveals new opportunities for the rapid transmission of data on the position of objects in near-Earth space, access to distributed data storages and input of thematic data arrays into international scientific turnover. At the same time, web-GIS is the most effective tool for distributed data processing and quick results presenting for a wide range of users [18].

Despite the fact that the attribute data of space objects is less changeable over time, it is very often fragmentary and irrelevant. To study the whole variety of bodies in near-Earth space, it is necessary to have the most complete set of data that forms the descriptive context of the constantly evolving situation in the NES. Especially this concerns information about the shape of the object, when there are no three-dimensional models of the surveyed SO. The shape and size of objects can be used in surveillance conditions modeling and to design terrestrial and orbital tracking devices.

The final goal of this research carried out at Moscow State University of Geodesy and Cartography (MIIGAiK) is to create a single aggregating geoinformation platform for nearEarth space modeling, including artificial and natural objects motion, the Earth's physical fields influence on them, remote sensing conditions modeling and planning, orbital parameters analysis and other tasks.

\section{Geoinformatics and near-Earth space: expanding the subject area}

\subsection{Regulatory issues and classification development}

Until now, there was no term of a near-Earth space geographic information system. At the same time, the existing legal, regulatory and technical documentation does not specify the development of geoinformation systems operating with three-dimensional spatial data, not to mention their temporal dynamics (the so-called "4D systems"):

- State Standard 34.003-90. Information technology. Set of standards for automated systems. Automated systems. Terms and definitions. 
- State Standard R 50828-95. Geoinformatic mapping. Spatial data, digital and electronic maps. General requirements.

- State Standard R 51605-2000. Digital topographic maps. General requirements.

- State Standard R 51606-2000. Digital topographic maps. Classifying and encoding system for digital cartographic information. General requirements.

- State Standard R 51607-2000. Digital topographic maps. Digital description rules for cartographic information. General requirements.

- State Standard R 51608-2000. Digital topographic maps. Quality requirements.

- State Standard R 52055-2003. Geoinformatic mapping. Spatial models of terrain. General requirements.

- State Standard R 52155-2003. Federal, regional, municipal geographical information systems. General technical requirements.

- State Standard R 52293-2004. Geoinformatic mapping. System of electronic maps. Electronic topographic maps. General requirements.

- State Standard R 52438-2005. Geographical information systems. Terms and definitions.

- State Standard R 52439-2005. Digital terrain models. Feature catalogue. Requirements for structure.

- State Standard R 52571-2006. Geographical information systems. Spatial data compatibility. General requirements.

- State Standard R 52572-2006. Geographical information systems. Coordinate frame. General requirements.

- State Standard R 52573-2006. Geographic information. Metadata.

- State Standard R 53339-2009. Framework data. General requirements.

- State Standard R ISO 19105-2003. Geographic information. Conformance and testing.

- State Standard R ISO 19113-2003. Geographic information. Quality principles.

The above-mentioned documents provide only information on the content and presentation of cartographic information, including digital, plane maps, description of the GIS requirements and structure to work with these materials. Also, the traditional classification of GIS by spatial coverage is tied to the Earth's surface. There is no indication that a spatial object can be a spacecraft or space debris fragment; only traditional objects located on the Earth's surface and processed by classical GIS are defined.

Many particular tasks are solved in different organizations dealing with monitoring and modeling of near-Earth space, while the interaction between them is often minimal. Moreover, access to the results of their research is often denied for other scientists. The available catalogs and databases often contain fragmentary, irrelevant content, considering space objects as point objects, and not as three-dimensional. There is a lack of public access to the results of space objects surveillance and specific software libraries and tools for NES state visualization in Russia. A comprehensive modeling of the current and future situation in the NES requires a software solution that will combine and aggregate data from various organizations of the global space industry. The prospects for raising the efficiency of SD control in near-Earth space and decision support based on the use of GIS technologies are obvious. Modern tools allow the development of a fully functional geographic information system that will leave behind the capabilities of desktop software solutions, created earlier.

In earlier studies [18], it was proposed to use GIS technologies, in particular, web-GIS solutions, to model near-Earth space and all objects in it as the most promising way for distributed data processing, using a near-Earth space geoinformation system (NES GIS) prototype, the subject area of which at the first sight was the Earth as a single entity, and the space from the upper boundary of the atmosphere to the orbit of the Moon, with all 
space objects located there, considered as three-dimensional objects of a specific shape. It is important to understand that near-Earth space environment is a complex system with internal structure and interactions. As a result, NES GIS differs from classical geographical information systems in its object content and tasks that are being solved.

Also it should be noted that acronym NES GIS is, first of all, an independent class of geoinformation systems, which is distinguished by us, combining various developments of other authors and organizations, and secondly - a self-titled prototype specifically developed at MIIGAiK. In terms of issue-related orientation, NES GIS can be classified as specialized systems since geoinformation modeling of NES is a very specific task at the current stage of geoinformatics development. They can be used as a support in education or solving administrative tasks (decision-making on satellites maneuvers, assessing and planning the possibility of the Earth's surface specific area remote sensing, etc.). According to the spatial coverage, the NES GIS should be classified as global on a scale of the Earth, although it does not use digital maps at a scale of 1: 4,000,000 and smaller, but uses a virtual digital globe, for which, by the way, in the Russian Federation there are no requirements for the classification, encoding and digital description of cartographic information.

In terms of their functionality, projects appertaining to the NES GIS class can develop in one of several directions, depending on the target group of users:

- GIS-builder - a full-featured GIS for spatial and attribute data input, editing and storage, as well as complex procedures for spatial analysis and geodata modeling, implemented with a built-in universal toolkit or application programming interface (API).

- GIS-analyst - geo-information analysis of data contained in spatial database (DB).

- GIS-viewer - viewing information that was previously inserted and structured by access rights.

\subsection{Technological search}

The research in the field of near-Earth space environmental modeling and development of relevant software in MIIGAiK began in early 2010s. At the beginning, general issues of creating a system as a part of a hardware and software tools set to provide space objects tracking in outer space have been discussed [19]. Such a system could synthesize threedimensional models of space objects based on two-dimensional images of a target object. Moreover, the grid computing technology was observed and a distributed network based on BOINC (The Berkeley Open Infrastructure for Network Computing) was set up [20]. At first it was designed for geomodeling, geoportal deployment and physical process simulation. But then a related project of space monitoring allowed redeploying of the network functionality.

After that the research was focused on algorithm analysis for modeling of 3D models of space objects [21], software solutions to monitor near-Earth space and motion simulation of SO. The first steps in researching the possibilities to develop the NES GIS began with a competitive analysis of the Systems Tool Kit (STK) software suite, developed by the American company Analytical Graphics, Inc. (AGI) and designed to perform a complex analysis of ground, air, sea and space-based forces by civil and military specialists: localization of objects in time and space, as well as their relative position determination among themselves, taking into account a number of external conditions (Fig. 1). Its weaknesses were the lack of technical support outside the United States, a weekly trial version only for registered users with personal identification (SIM card bought in the US) and a high final cost (65 thousand US dollars in 2014), excluding software modules for the Earth remote sensing (RSE) payload simulation and other additional units. 


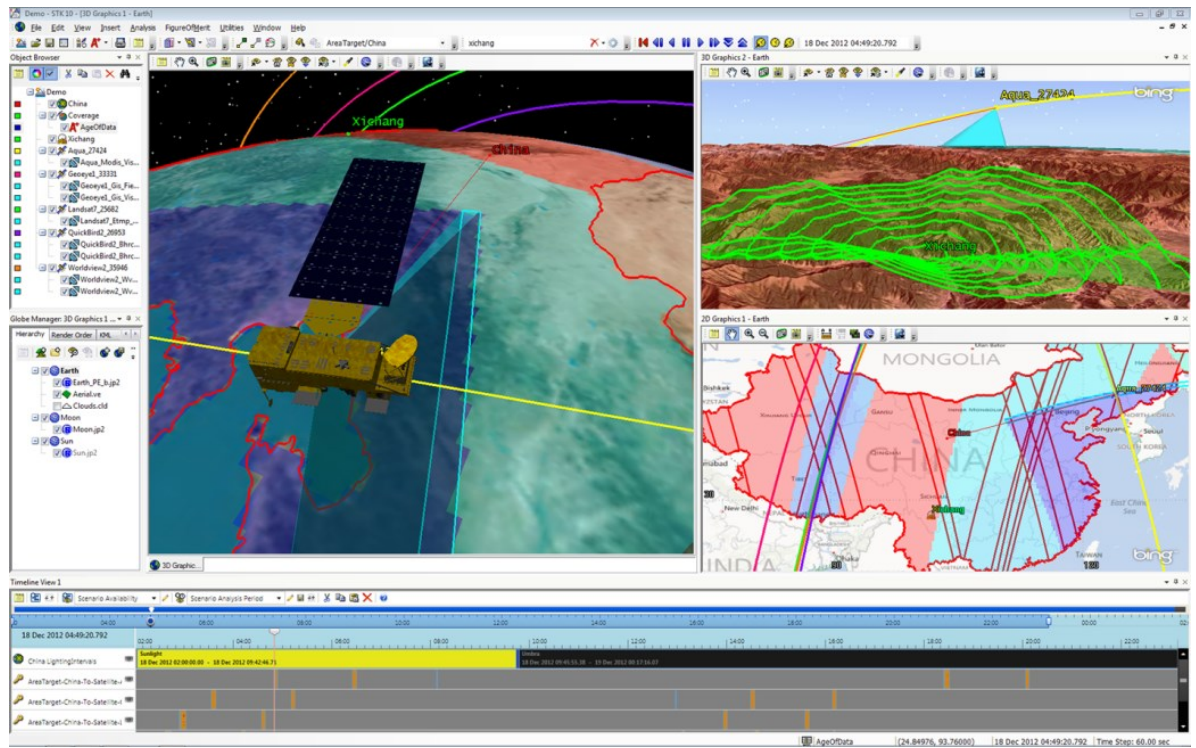

Fig. 1. The Systems Tool Kit software suit's workspace components [22].

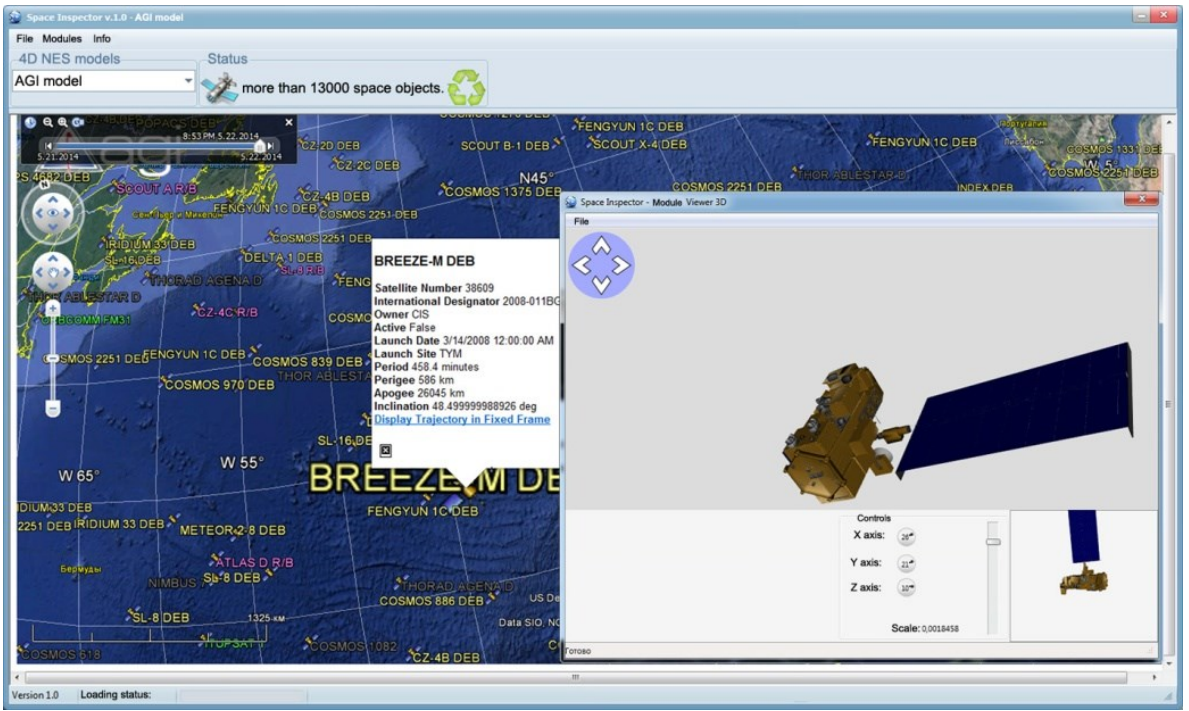

Fig. 2. The system Space Inspector v.1.0 based on Google Earth API was developed in MIIGAiK. In front - a software module Viewer3D for space objects visualisation is presented. On the background a main window with pre-loaded space objects database provided by Analytical Graphics Inc. [18].

The first prototype of our own software - Space Inspector was a desktop modular solution based on the Google Earth API and consisting of a head program loading web content with a connected SO database (in . $\mathrm{kml}$ format) and a subsystem viewing threedimensional models (Fig. 2). Its functional features included such capabilities as: space object position visualization from open databases, using a three-dimensional scene in the WGS 84; space objects 3D models display in the formats .obj, .ase, .dae (COLLADA); NES event scripts visualization. Due to the properties of the Embarcadero RAD Studio XE4 IDE, the main module was created using the VCL library and executed by the computer's central processor, while the Viewer3D module was created with the 
FireMonkey platform working on the user's graphics processor unit. Google Earth API required a pre-installed plug-in on the user's computer, which, together with the abovementioned weak points, made the system unattractive for the end user, both in terms of working with resources and in terms of using third-party software solutions. Moreover, only the Windows operating system was the working platform of this prototype. That's why it was decided to refuse this concept in favor of a web-GIS solution.

In 2015, the technical support for Google Earth API was over, which put a full stop to the development of the first version of the NES GIS. The search for alternatives led to the WebGL Globe project. However, the difficulty of working with spatial data and the lack of built-in mathematical tools for processing them were its disadvantages. The open-source graphics library named Cesium was chosen after technological search as the basis for the second version of the software suite for spatial data processing and space objects position visualization, which uses a graphics processor and has a number of advantages compared with other existing software tools for web-GIS development [23]. Using this tool, one can perform high-precision calculations and mathematical processing of different coordinate and reference systems: conversion from geographic (latitude, longitude, altitude) to Cartesian coordinates; fast processing of Cartesian, spherical, map coordinates, matrices and quaternions; work with Julian date, leap seconds, and time standards such as Coordinated Universal Time (UTC) and International Atomic Time (TAI).

\subsection{Update of near-Earth space GIS term}

Analysis of the spacecrafts' orbits, including orbital astrophysical observatories, as well as a scientific discussion with colleagues, gave the idea of the need to expand the near-Earth space modeling boundaries. It became obvious that the Earth's satellites can appear in its gravitational field not only within the average distance to the orbit of the Moon. The Earth, like any other celestial body, has three gravitational spheres (gravispheres), in order of their radius increase: a sphere of attraction, a sphere of influence and the Hill sphere [24]. Let's denote the radius of a sphere of attraction as $R_{A}$, the radius of a sphere of influence as $R_{I}$ and the radius of the Hill sphere as $\mathrm{R}_{\mathrm{H}}$.

Hill sphere is a sphere of gravitational influence around an astronomical object, located between the Lagrange points $L_{1}$ and $L_{2}$, in which it is able to hold its satellite, despite the attraction of the object (the more massive body), around which it orbits (centripetal force, i.e. the sum of three forces: the forces of attraction (gravity) from the superior and inferior bodies, as well as the centrifugal force acting on the object at a given point, directed towards the inferior body). Hill sphere radius is calculated using the expression:

$$
R_{H} \approx a \cdot \sqrt[3]{\frac{m}{3(M+m)}} \approx a \cdot\left(\frac{\frac{1}{3} \cdot m}{M}\right)^{\frac{1}{3}}
$$

where $a$-semi-major axis of the inferior body's orbit, $m$ and $M$ - masses of the inferior (for example, the Earth) and the superior body (for example, the Sun), while $m \ll M$. If the inferior body's orbit eccentricity $e$ does not tend to zero (cannot be ignored), then the expression (1) takes the form:

$$
R_{H} \approx a(1-e) \cdot \sqrt[3]{\frac{m}{3(M+m)}}
$$

The values of the gravispheres' radii for the terrestrial planets and the Moon are presented in Table 1. 
Table 1. The terrestrial planets' gravispheres mean values [25].

\begin{tabular}{|c|c|c|c|}
\hline $\begin{array}{c}\text { Celestial } \\
\text { body }\end{array}$ & $\boldsymbol{R}_{\boldsymbol{H}}(\mathbf{k m})$ & $\boldsymbol{R}_{\boldsymbol{I}}(\mathbf{k m})$ & $\boldsymbol{R}_{\boldsymbol{A}}(\mathbf{k m})$ \\
\hline Mercury & 221321 & 112000 & 24000 \\
\hline Venus & 1011150 & 616000 & 69000 \\
\hline Earth & 1496574 & 929000 & 260000 \\
\hline Moon & 59000 & 56000 & 13000 \\
\hline Mars & 1084073 & 578000 & 128000 \\
\hline
\end{tabular}

A scheme of the Earth's gravitational spheres is shown in Fig. 3. Nevertheless, one should not forget that the Moon also has its own gravispheres, which intersect with the Earth's gravitational spheres for certain. Once in them, the body is under the influence of the Earth's natural satellite, tending to fall on its surface.

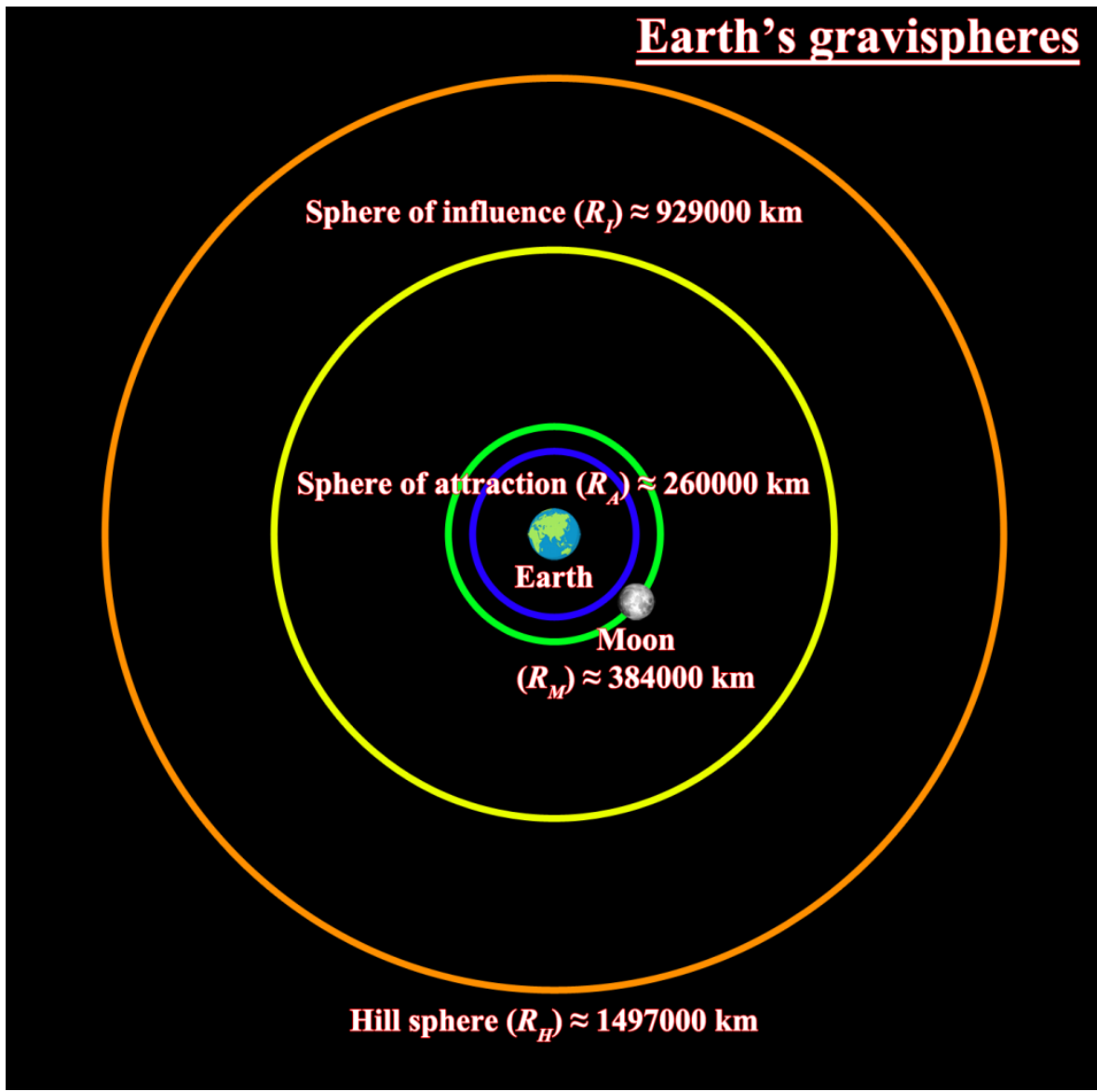

Fig. 3. The Earth's gravispheres scheme.

To sum up, an independent class of near-Earth space geoinformation systems is distinguished, which differ in their subject area - the Earth as a single entity and its environment within the Hill sphere (centered in the Earth), full of natural and artificial space objects in constant motion, and designed to work with space objects spatial and attribute data, which is fundamentally different from classical GIS. Expanding the limits of NES geoinformation modeling, as well as the fact of the Earth's physical fields influence on the spacecrafts trajectories, including remote sensing, the operation of navigation 
systems, the results of geodetic measurements and geophysical researches, make it possible and reasonable to perform geoinformation modeling of these fields within the framework of prospective GIS for the purpose of appropriate quantitative assessments.

\subsection{Comparison and integral estimation of GIS development tools, databases and services for modeling of space objects motion}

Earlier in [18], an analysis of tools that can be used in the NES GIS development was carried out. The comparison was made based on the functionality, operating principles and commercial features of the Cesium library, Google Earth API, and Google Maps API. At the same time, the Cesium library turned out to be the preferred development tool for the goal set by the authors.

The closure of a number of databases and services led to a reduction in the volume of information available on the subject of near-Earth space's state modeling. As a result, the database of The Union of Concerned Scientists (UCS) has become the main source of information about objects in near-Earth space [26]. However, it contains only information on active spacecrafts (still in use). In addition, SO are considered as point objects, because there is no descriptive information about their size and shape. The work with it is complicated as it's designed in the spreadsheet editor. In [9], the comparative analysis of databases and services for modeling of man-made space objects motion in NES was updated: the DISCOS database of the European Space Agency was added as a reference, the Swiss Solenix service based on the Google Earth API was excluded, and the UCS database was added. [27]:

For an integral estimation of databases and services efficiency, expression (3) was used

$$
Q_{e f f}=\eta_{d c} \times \eta_{u} \times \eta_{s p}
$$

where $\eta_{d c}$ - completeness of data property, $\eta_{u}$ - data update property, $\eta_{s p}$ - property characterizing the software platform underlying a database or service (software efficiency). During the comparison of databases / services, 90 categories of information about space objects were used to assess the completeness of data property, including orbital parameters, data on current location and motion, launch and destruction data, launch vehicles and spaceports, etc. The data refresh rate was marked out as a separate category, characterizing by the data refresh property. It should be noted that the slowest solution was the "What's Up" database, where the location of SO was updated once a day (or 86400 seconds) [28], which determined the corresponding threshold value. Potential development tools analysis for NES GIS in 15 categories identified their software platforms efficiency properties. To find out the properties of data completeness, data update and software platform efficiency, expression (4) was used [27]:

$$
\eta_{x}=\frac{\sigma_{t}-\sigma_{a}}{\sigma_{t}+\sigma_{a}}
$$

where $\sigma_{t}$-parameter's threshold value defining the desired property, $\sigma_{a}$ - parameter's actual value defining the desired property.

The results are presented in Table 2 .

The final efficiency assessment, in case of the DISCOS database selection as a source for the NES GIS data input, ranks over the closest competitor by 3,5 times. DISCOS significantly trump the UCS database [18], which was previously selected as a reference DB in terms of information content. Both current and future solutions based on the Cesium

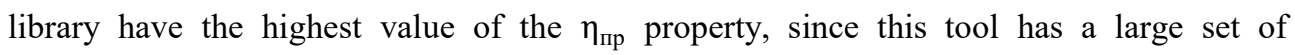
capabilities necessary for creating the NES GIS, including a three-dimensional scene, time dynamics and a mathematical apparatus for processing spatial data. 
Table 2. The results of space objects' databases and modeling services integral assessment [9].

\begin{tabular}{|c|c|c|c|c|c|c|c|}
\hline \multirow{2}{*}{ Property } & \multicolumn{5}{|c|}{ Database } & \multicolumn{3}{c|}{ Service } \\
\cline { 2 - 9 } & DISCOS & $\begin{array}{c}\text { "What's } \\
\text { Up" }\end{array}$ & AGI & UCS & N2YO & SpaceBook & iSat \\
\hline $\begin{array}{c}\text { Completeness } \\
\text { of data } \eta_{d c}\end{array}$ & 0,593 & 0,169 & 0,078 & 0,169 & 0,132 & 0,047 & 0,04 \\
\hline Data update $\eta_{u}$ & 0,999 & 0 & 0,999 & 0,999 & 0,999 & 0,999 & 0,999 \\
\hline $\begin{array}{c}\text { Software } \\
\text { efficiency } \eta_{s p}\end{array}$ & 0,765 & 0,2 & 0,2 & 0,765 & 0,25 & 0,765 & 0,765 \\
\hline $\begin{array}{c}\text { Integral } \\
\text { criterion } Q_{e f f}\end{array}$ & 0,453 & 0 & 0,016 & 0,129 & 0,033 & 0,036 & 0,03 \\
\hline
\end{tabular}

\subsection{Software development}

At the moment, a prototype of a conceptual NES GIS has been developed (Fig. 4) with an accompanying software tool for prediction of SO location and three-dimensional dynamic scenes generation, which makes it possible to calculate the motion of satellites in the nearEarth space at a time specified by the user within the forecast script, to display attribute data and to evaluate the possibility of getting imagery by Earth remote sensing satellites, in particular, for cross-calibration of the Earth's remote sensing payload [29]. This GIS, being developed as an open system, is designed to combine and aggregate the results of nearEarth space organizations' studies.

Despite the large number of models, methods and theories for predicting the motion of space objects, constituted three large groups - analytical, numerical and semi-analytical, only the analytical SGP4 model (after 2006 - the combined SGP4/SDP4 model) has input data for calculations as well as the necessary documentation and program code [30]. The orbital parameters for this model are published in the format of a Two-Line Element set, also known as TLE. It includes both the classical elements of Kepler's orbit and those specific to the SGP4 model [31]. Up-to-date data on the most significant spacecrafts and space debris fragments, as well as some of the archived TLE sets (for example, for the Soviet station Salyut-7) are always available without the need for registration and SMS verification on the portal of Dr. Thomas Sean Kelso [32]. Historical data files, as well as current orbital parameters for all cataloged objects (by American specialists) are available for registered users on the special portal of the US Space Command (USSPACECOM, formerly known as NORAD) [33]. One should also remember that the final product of this model is the position and velocity vectors in the TEME (True Equator and Mean Equinox) reference frame - a type of Earth centered inertial system, which is a coordinate system formed by the projection of the mean equinox of date onto the true equator of date [34]. As for the Russian developments, for example, the information bulletins of JSC "Vimpel Interstate Corporation" contain ephemerides of space debris objects in GEO and HEO, but restrictions on their application and presentation are imposed, as well as there is no description of the numerical model for developers and the program code, in contrast to SGP4. According to the organization [35], these ephemerides are osculating Keplerian elements in the inertial coordinate system assigned to the J2000 epoch. The orbital parameters are obtained using a numerical model of motion that takes into account the perturbation from the Earth's gravitational field (all harmonics of the geopotential up to and including the $8^{\text {th }}$ ), the gravitational fields of the Moon and the Sun (as point masses with coordinates as given in the DE-405 model), the Earth's atmosphere (as given in the State 
Standard R 25645.166-2004. Earth upper atmosphere. Density model for ballistic support of flights of artificial earth satellites), the pressure of the direct radiation flow from the Sun.

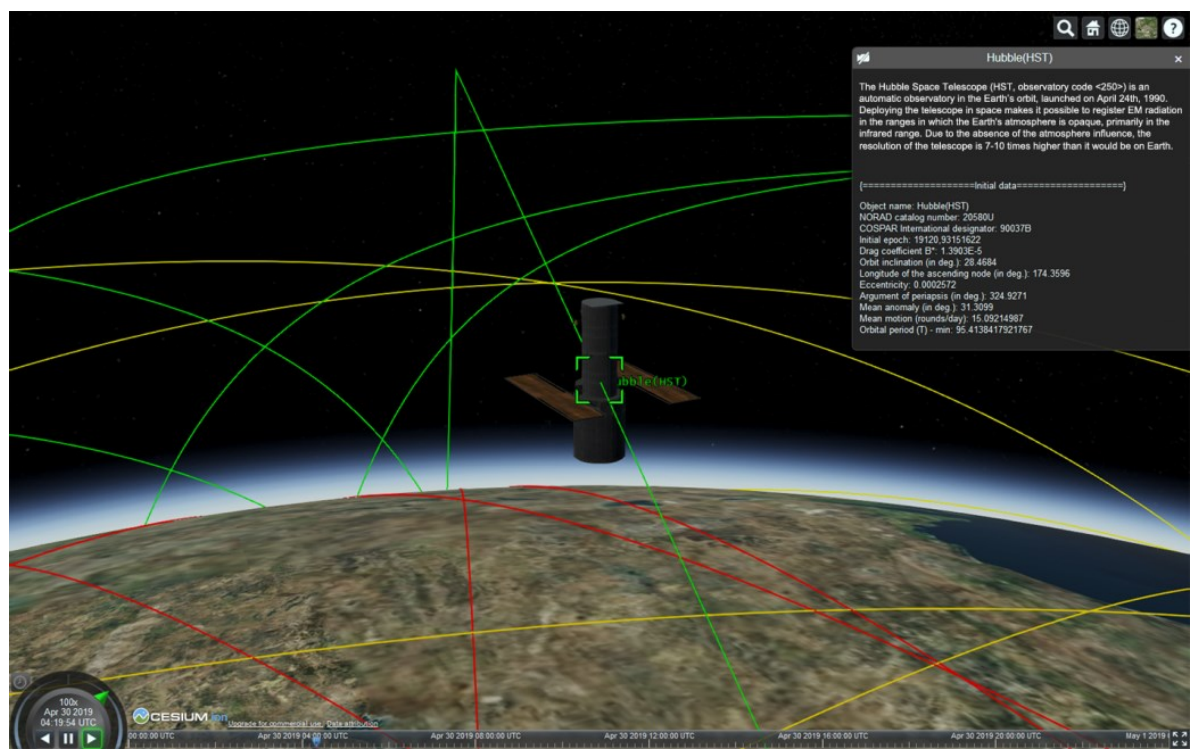

Fig. 4. Visualization of the Hubble Space Telescope 3D model, orbits and ground tracks using the near-Earth space GIS prototype (30.04.2019, 00:00:00 (UTC) - 23:59:30 (UTC)).

As a tool related to the NES GIS, the above-mentioned TLEPropagator software was developed [23], which is necessary to perform predictions of satellite location using TLE for the combined SGP4/SDP4 model, and to generate 3D dynamic scenes files. In addition to the orbital parameters, the estimated time (taken from the initial epoch of the TLE set by default, but it can be changed by the end user), the coordinate system, the number of calculated positions of the space object and the time step (in seconds) between the calculated positions are used as input data. TLEPropagator allows one to extract the contents of a TLE and calculate the state vector (position and velocity) of an object at a given moment in time, or for a time interval with a given step.

It should be noted that a three-dimensional dynamic scene is a script (in .czml format) containing coordinates of space objects with a time reference (orbits and/or tracks), their 3D models (if necessary, in .glTF format), intersection points with an object of interest, as well as text descriptions, processed by the mathematical core of NES GIS for a following view to the user [36, 37]. Fig. 5 shows a three-dimensional dynamic scene of a TESS space telescope mission to search exoplanets in the time interval from May $5^{\text {th }}, 2018,00: 00: 00$ (UTC) to May $16^{\text {th }}, 2018,23: 59: 30$ (UTC). A 3D model of the spacecraft in .glTF format at a scale of 1:1 is loaded. During the playback of the scene (as well as with the help of the coordinates and time stamps stored in the script), one can get the moments of studied SO perigee and apogee passage, its illumination by solar radiation and visibility from the Earth. It is also possible to catch up on the description of the object, the initial orbital parameters and the orbital period, and thanks to the presence of a 3D model, its shape and structural features. 


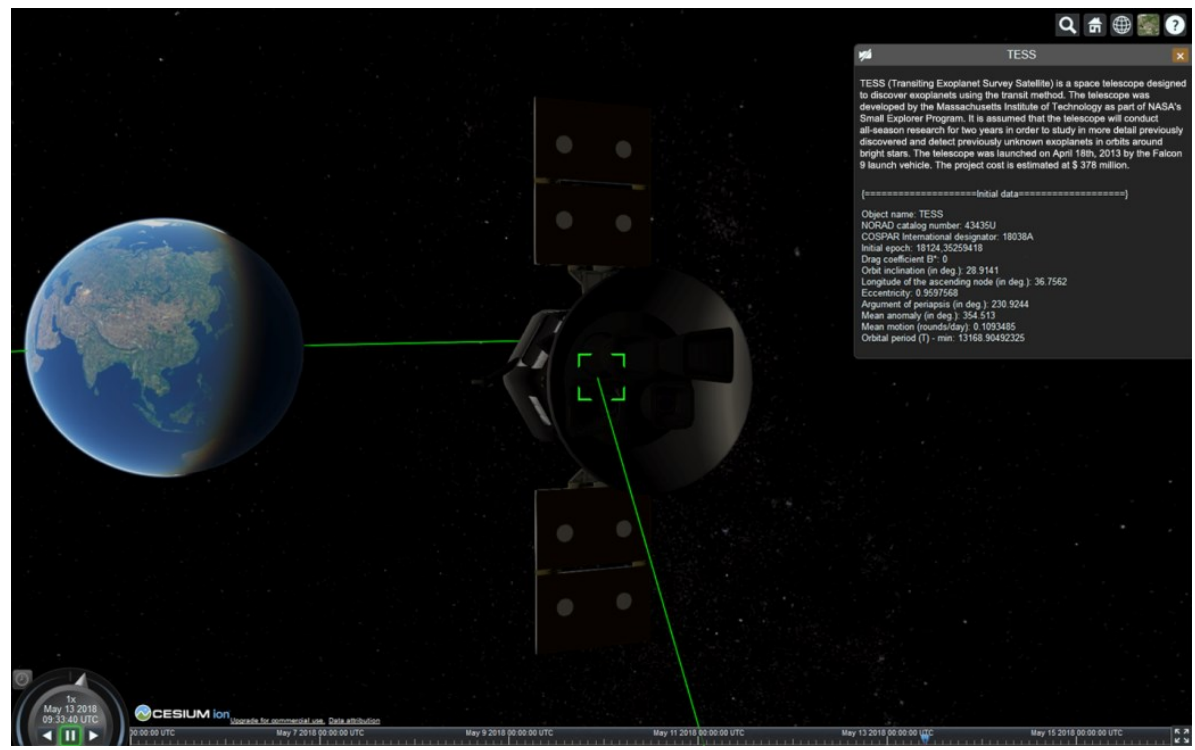

Fig. 5. Visualization of the TESS telescope 3D model and orbit using the near-Earth space GIS prototype (05.05.2018, 00:00:00 (UTC) - 15.05.2018, 23:59:30 (UTC)).

On the proposal of "VNIIEM Corporation" JC, the TLEPropagator tool was updated for the purpose of mathematical and algorithmic support of activities for cross-calibration of Kanopus-V spacecraft constellation's sensors. In this regard the output of prediction information was changed, specifically: the projection of the studied spacecrafts' orbits for the required time intervals, i.e. ground tracks are automatically written to a .kml file. An algorithm to search for intersections of RSE satellites ground tracks was developed, which makes it possible to find areas of the Earth's surface observed from the satellites participating in the calibration with a time difference of no more than 30 minutes and up to 15 degrees deviation from nadir for the line of sight [29]. The result of the algorithm are exported in the. $\mathrm{kml}$ format ground tracks of the involved satellites, as well as the ground tracks' points of intersection, with a description of the points passage moments by remote sensing spacecrafts and the proximity to the radiometric calibration polygons (optional).

\subsection{Experimental results and prospects}

As part of experiments with the upgraded TLEPropagator software tool, the execution time for the space object prediction and the volume of resulting data, depending on the prediction interval and the time step, was measured [18]. The estimation was carried out both for the. $\mathrm{kml}$ file containing the SO ground track and for the .czml file containing the $\mathrm{SO}$ orbit. The experiment was performed on a personal computer (PC) with the following characteristics:

- CPU: AMD Phenom II X6 1090T, $3500 \mathrm{MHz}$;

- Motherboard: Asus M2N68-CM;

- $\quad$ RAM: 8192 Mbytes (DDR2-800 DDR2 SDRAM);

- GPU: NVIDIA GeForce GTX 550 Ti (1 Gbyte).

The predictions were made for 1, 3 and 7 days, with a time step from 1 second to 1 minute, which determined the number of predicted points. Due to the features of the SGP4/SDP4 analytical model, for each moment in time (predicted location in NES) within the prediction span, 25 parameters are formed at once, including position and velocity vectors, latitude and longitude, current inclination, orbit's eccentricity and other orbital 
parameters. As a result, the execution time of the predicted orbit and ground track points is the same. The obtained results are presented in Table 3.

Table 3. The results of remote sensing satellite ground track and orbit figures evaluation [18].

\begin{tabular}{|c|c|c|c|c|c|}
\hline $\begin{array}{c}\text { Prediction } \\
\text { span (days) }\end{array}$ & $\begin{array}{c}\text { Prediction step } \\
\text { (sec) }\end{array}$ & $\begin{array}{c}\text { \# of predicted } \\
\text { points (units) }\end{array}$ & $\begin{array}{c}\text { Execution } \\
\text { time (sec) }\end{array}$ & $\begin{array}{c}\text { Ground } \\
\text { track data } \\
\text { size (Mbyte) }\end{array}$ & $\begin{array}{c}\text { Orbit } \\
\text { data size } \\
\text { (Mbyte) }\end{array}$ \\
\hline \multirow{4}{*}{1} & 60 & 1440 & 0,437 & 0,049 & 0,087 \\
\cline { 2 - 6 } & 20 & 4320 & 1,25 & 0,147 & 0,26 \\
\cline { 2 - 6 } & 10 & 8640 & 2,5 & 0,295 & 0,52 \\
\cline { 2 - 6 } & 5 & 17280 & 4,99 & 0,59 & 1,04 \\
\hline \multirow{4}{*}{3} & 1 & 86400 & 24,88 & 2,95 & 5,20 \\
\cline { 2 - 6 } & 60 & 4320 & 1,26 & 0,149 & 0,26 \\
\cline { 2 - 6 } & 20 & 12960 & 3,73 & 0,442 & 0,782 \\
\cline { 2 - 6 } & 10 & 25920 & 7,46 & 0,887 & 1,56 \\
\hline \multirow{4}{*}{7} & 5 & 51840 & 15,07 & 1,78 & 3,12 \\
\cline { 2 - 6 } & 1 & 259200 & 75,04 & 8,85 & 15,6 \\
\cline { 2 - 6 } & 20 & 10080 & 2,9 & 0,349 & 0,616 \\
\cline { 2 - 6 } & 10 & 30240 & 8,69 & 1,03 & 1,85 \\
\cline { 2 - 6 } & 5 & 60480 & 17,39 & 2,06 & 3,7 \\
\hline & 1 & 120960 & 34,77 & 4,13 & 7,39 \\
\hline
\end{tabular}

It was also defined that the volume of data on the intersection points is much less than the volume of data on the SO ground tracks and orbits. 19 intersections with crosscalibration polygons were obtained on the weekly prediction for the Kanopus-V spacecraft No. 6, which is 8,53 Kbytes. At the same time, 563 intersections with Landsat 7 and Landsat 8, Sentinel-2A and Sentinel-2B satellites ground tracks were obtained on the weekly prediction for Kanopus-V No. 5 and Kanopus-V No. 6, which is 205 Kbytes.

Extrapolating the results obtained above, it will take 40,23 days for a weekly prediction of 20,000 space objects location, running on a single PC with similar characteristics to the machine used for the test. In this case, only the orbit data size will be 722,07 Gbytes (ground tracks - 403,32 Gbytes). These results provide a direct evidence of the need to use distributed data processing and motion prediction of all cataloged space objects [38].

For the SGP4/SDP4 analytical model a series of experiments were done to estimate the accuracy of predicted location using the reference data. The tracks of the Kanopus-V-IK RSE satellite, obtained from an onboard GPS/GLONASS receiver with an accuracy of 10 meters in an absolute coordinate system with a WGS 84 datum, were taken as reference coordinates. The assessment technique consisted of the following stages:

- Satellite location prediction based on current TLE ephemerides for the time specified in the GPS track file using TLEPropagator.

- Conversion of predicted data from the TEME inertial reference frame into a Cartesian Earth centered coordinate system with a fixed reference frame (known as ECEF).

- Main accuracy indicators estimation for the time a chosen satellite passes the selected part of the orbit (GPS track comparison).

It is important to remember that when converting from TEME to ECEF, one need to take into account: the difference between Universal Time (UT1) and Coordinated Universal Time (UTC), UTC and International Atomic Time (TAI), TAI and Terrestrial Time (TT), as well as the influence of polar motion along the $\mathrm{X}$ and $\mathrm{Y}$ axes $[39,40]$.

For the track with reference coordinates characterizing the spatial location of KanopusV-IK on September $14^{\text {th }}, 2017$, at a time interval of 08:40:53 - 08:42:33 UTC, two TLE sets were taken with an initial epoch of September $14^{\text {th }}, 2017$, 08:56:40 UTC (a little later 
than the reference track, a drag coefficient $\left.B^{*}=0,000023855\right)$ and September $13^{\text {th }}, 2017$, 12:23:51 UTC (almost 20 hours before the reference track, a drag coefficient $B^{*}=$ 0,000032374 ). Table 4 shows the results of SGP4/SDP4 model accuracy estimation, based on two sets of ephemerides and 11 reference points from an onboard receiver with a time step of 10 seconds. A measurement error was calculated at confidence level of $90 \%$. SD denotes a standard deviation.

Table 4. The results of SGP4 model's precision evaluation based on GPS reference data.

\begin{tabular}{|c|c|c|c|c|c|c|}
\hline \multirow{2}{*}{ TLE set } & \multirow{2}{*}{$\begin{array}{c}\text { GPS } \\
\text { points }\end{array}$} & Coordinates & $\begin{array}{c}\text { Mean } \\
\text { difference, } \mathbf{m}\end{array}$ & SD, m & $\begin{array}{c}\text { Standard } \\
\text { error, } \mathbf{m}\end{array}$ & $\begin{array}{c}\text { Measurement } \\
\text { error, m }\end{array}$ \\
\hline \multirow{3}{*}{14.09 .17} & \multirow{3}{*}{11} & $\mathrm{X}$ & 563,278 & 14,068 & 4,242 & 6,978 \\
\cline { 3 - 7 } & & $\mathrm{Y}$ & 751,070 & 20,883 & 6,296 & 10,357 \\
\cline { 3 - 7 } & & $\mathrm{Z}$ & 586,365 & 32,950 & 9,935 & 16,343 \\
\hline \multirow{3}{*}{13.09 .17} & \multirow{2}{*}{11} & $\mathrm{XYZ}$ & 1107,59 & 3,903 & 1,177 & 1,936 \\
\cline { 3 - 7 } & & $\mathrm{X}$ & 593,989 & 14,329 & 4,320 & 7,107 \\
\cline { 3 - 7 } & & $\mathrm{Z}$ & 761,024 & 22,287 & 6,720 & 11,054 \\
\cline { 3 - 7 } & & $\mathrm{XYZ}$ & 1163,99 & 1,411 & 0,425 & 0,700 \\
\hline
\end{tabular}

For the first track, the mean (spatial) difference (in ECEF) was 1,1 km, and the standard deviation was $0,0039 \mathrm{~km}$. For the second track, the average error was $1,16 \mathrm{~km}$, and the SD was $0,0014 \mathrm{~km}$. By this means, the result of the SGP4/SDP4 accuracy evaluation corresponds to the values stated in the literature and fit in the range from 1 to $2 \mathrm{~km} \mathrm{[41].} \mathrm{It}$ is a good idea to use a larger dataset for such an estimation and check the possibility of accuracy loss when converting from one coordinate system to another, as well as performing a long-term prediction, since the same work [41] notes that the actual value of the drag coefficient $B^{*}$ may differ from the initial one by one order of magnitude after 7-10 days. The adequate accuracy is achievable with a prediction for 2 or 3 days, due to a change in the atmosphere state, characterized in the TLE sets by the parameter $B^{*}$.

The visualization of geophysical fields in GIS is poorly developed at the moment. There is no method to visualize such parameters as the magnitude and direction of the gravity vector or any other geophysical field simultaneously in modern geographical information systems. Methods that have come from cartography are widely used to display geophysical fields, for example, contour lines or visualization with color scales, while fields such as geomagnetic and gravity are often considered as scalar, being in fact vector ones. In order to improve the Earth's gravity field data usage efficiency, the NES GIS prototype uses a special method designed to visualize vector fields on a plane using color, which allows it to give information about both the magnitude and direction of the gravity vectors at each point [42].

A geoportal prototype to display the results of near-Earth space and its components modeling uses the Cesium library for two-dimensional and three-dimensional data visualization (Fig. 6). It is hosted on a free web server provided by the Glitch service with Node.js installed [43]. 


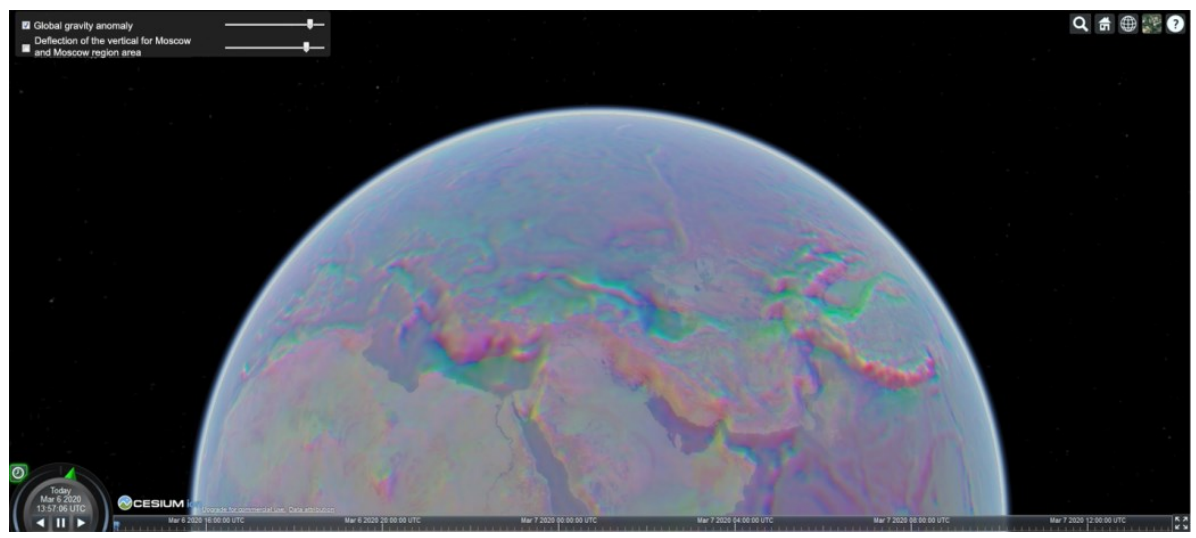

Fig. 6. Visualization of the global gravity anomaly using the near-Earth space GIS prototype.

The application of geoinformation modeling is also possible to support laser ranging activities performed by space and ground facilities. Laser ranging is necessary for the Earth surface fixed points coordinates determination (geodesy), as well as to calculate the present position and motion parameters of dynamic objects - navigation. In addition, it serves as a reference for calibration and verification of radio-technical systems and onboard time synchronization of the Russian GLONASS [44]. With the help of geoinformation modeling via the prospective NES GIS, the optimized distribution simulation of the laser ranging network is possible not only over the territory of the Russian Federation, but also throughout the world: each station can have a set of pre-defined parameters, including its spatial coordinates, visibility zone, interaction with other stations, etc.

Fig. 7 shows the Russian measuring network of satellite laser ranging (SLR) stations. Furthermore, the orbit and ground track of the Larets laser target satellite (also known from its launch vehicle as Rubin 4/r) on March 15 ${ }^{\text {th }}, 2019,00: 00: 00$ (UTC) and up to March $15^{\text {th }}$, 2019, 23:59:50 (UTC) are presented. The legend shows the SLR stations classes and their organizational ownership. Each station has a text description indicating the nearby settlement. The application of geoinformation modeling is worth for target satellites passage time stamps evaluation in the visibility zone of the Russian and international segments of SLR network, including the promising BLITS-M-1 and 2 spacecrafts, as well as other satellite systems. GIS modeling may also be important for lunar laser ranging tasks solving: it can be used to assess the availability of lunar retroreflectors, both for groundbased and for orbital laser tracking devices. In addition, it is possible to plan new retroreflective means deployment on the Moon, as well as to model promising constellations of laser communication satellites. 


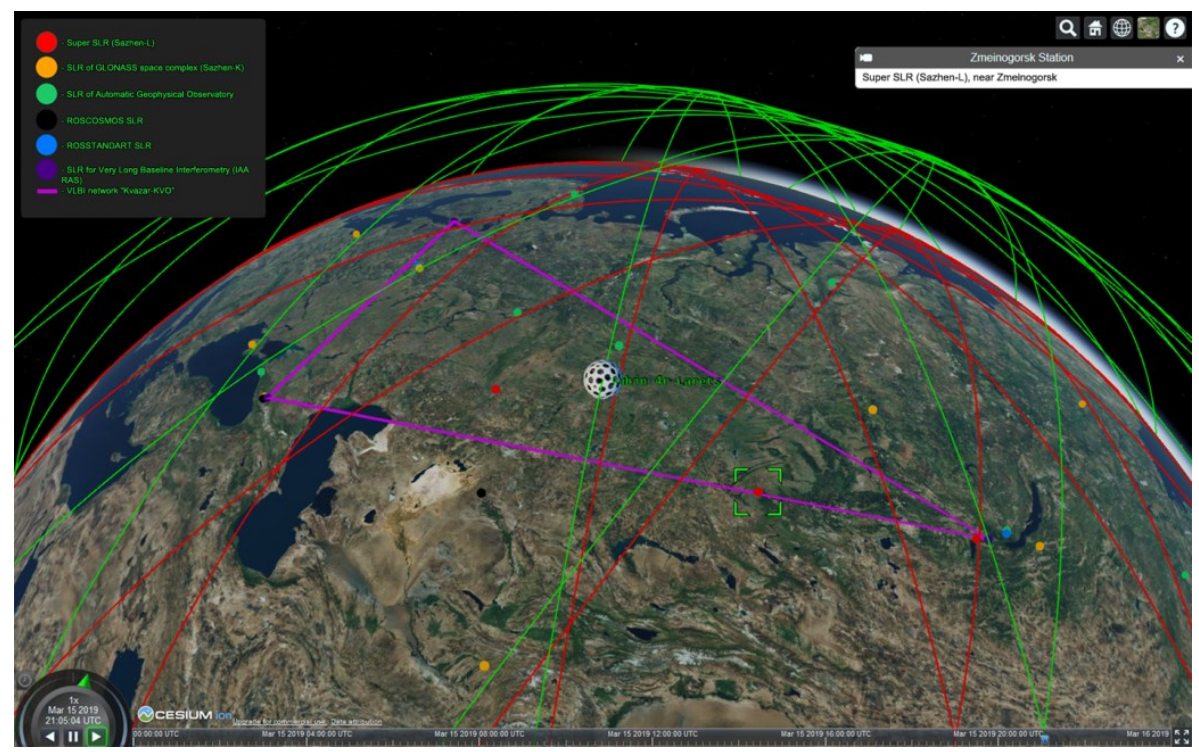

Fig. 7. Application of geoinformation modeling using the near-Earth space GIS prototype for the needs of laser ranging.

The project of the European Space Agency (ESA) DISCOS, which participated in the integral estimation and received the highest value of the data completeness property, does not have its own modules for spatial data visualization and NES location prediction. Moreover, the shape of space objects defined with text data is difficult to understand for the end user and is practically useless without information about their orientation. However, the authorized users of this system have access to the DISCOSweb API for DB queries generation. Hence, to fill the NES GIS with thematic data, it was decided to develop DISCOSParser - a software tool for space objects data extraction, structuring and storage, specifically [9]: general information on SO, data on their destruction, launches and launching sites data, rocket launchers and their stages, as well as the initial and decay orbit parameters (Fig. 8). With this tool, the user can make multi-parameter queries for single objects and their groups. The DISCOSParser user interface is designed to facilitate the work with the database, and the content categories translation into Russian with the relevant comments allows an ordinary user to better understand the thematic content without specific knowledge. The application of the DISCOSweb API allows preparing a JSON structure of different content for the selected SO from a compound query and save the needed information without unnecessary time loses, which is also convenient for data input into a single aggregating web-GIS of near-Earth space. This software tool continues to update: the possibility of obtaining TLE data with the COSPAR ID is considered right now in order to generate small 3D scenes (simplest visualizations) in the program window, explaining the orbital parameters of the selected space objects. 


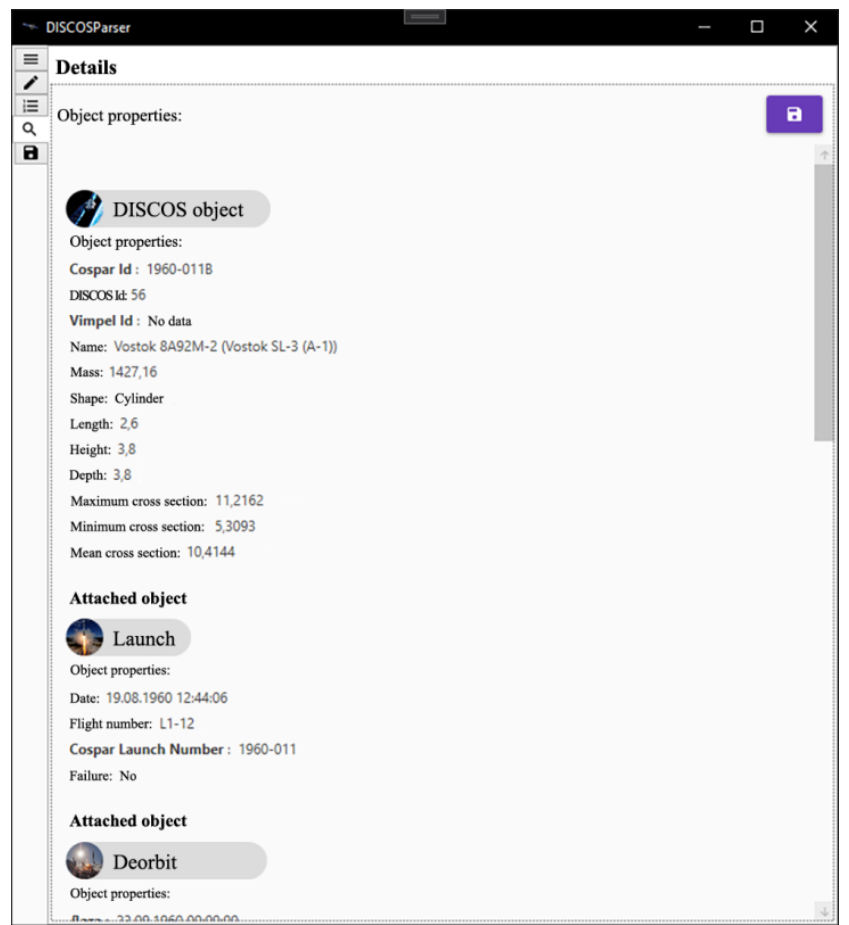

Fig. 8. Vostok (8K72) L1-10 launch vehicle data received by DISCOSParser software tool prototype [9].

\section{Results and discussion}

The main result of this research is an introduction of a new independent class of GIS, subject area of which is the Earth as a single entity, and the space within the Hill sphere, with all natural and artificial space objects located there in constant motion, considered as $3 \mathrm{D}$ rather than point objects. This class can be referred to as near-Earth space GIS and used to perform information, reference, administrative and other functions in form of primitive GIS viewers, advanced GIS analysts and full-featured GIS builders. The Earth's physical fields should also appear as objects of NES geoinformation modeling.

However, current existing legal and regulatory acts do not outline the development of GIS working with 3D spatial data, not to mention their temporal dynamics. The traditional classification of geographical information systems by spatial coverage is related to the Earth's surface. Spacecrafts or space debris fragments are not defined as spatial objects, while only terrestrial objects located on the surface are objects of classical GIS modeling.

The Cesium open-source graphics library was chosen as the basis of the developed MIIGAiK's prototype for spatial data processing and space objects location visualization, since it uses a GPU and has a number of advantages over other existing web-GIS software development tools. Obviously, it is necessary to create an open geo-information software environment for NES modeling and control, combining a set of geospatial data, aggregating the results of the space industry participants' activities and providing the generation of object-oriented models of space objects in statics and dynamics based on the platform of a specialized thematic web-oriented GIS with access via a geoportal.

Based on the results of the integral estimation, a reasonable solution seems to be the application of data from the DISCOS DB using a single aggregating near-Earth space webGIS, combining and adjusting fragmented information from various sources, including the 
ESA project, about objects, occurrences and events in near-Earth space, with the purpose of new data obtaining and hidden patterns identification. The current location data should be formed with several propagation models, but at the moment the analytical SGP4/SDP4 model is the only available one, which declared accuracy was verified using the reference data of the Kanopus-V-IK GPS receiver and reached slightly more than $1 \mathrm{~km}$ during the first day. Also, it is necessary to provide an additional table with the spacecrafts payload characteristics in the database of the NES GIS prototype, since there are still no public DB of this kind [17]. The TLEPropogator software tool test results pointed out that it is necessary to use distributed processing and motion predicting assets when working with a large number of space objects.

Since there are no open geoinformation software solutions in Russia for a complex analysis of miscellaneous data on near-Earth space objects coming from various organizations, and there is no organization that provides such activities, the development of such a single aggregating geoinformation platform is relevant and valid task that promotes the development of the space services market.

\section{Conclusion}

Any scientific sphere requires an information system for information collection from various sources, its verification and structuring, knowledge extraction, complex analysis and systematic research implementation [45], and the field of the near-Earth space state modeling is not an exception. Nevertheless, the NES GIS may also be required for solving a number of practical (industry) tasks and services, including those provided by satellite operators and developers, for example:

- $\quad$ satellite NES motion modeling within the prediction script;

- natural and artificial SO control and record;

- geophysical fields and their anomalies modeling (including volume and directional data; for example, the Earth's radiation belt dynamics modeling for space missions planning);

- application of developed method designed to visualize Earth's vector fields on a plane using color;

- diverse and comprehensive attribute, geometric and position data display, as well as three-dimensional models of selected space objects;

- survey time calculation of the Earth's surface area by a RSE satellite with the given parameters of sensors, taking into account the current meteorological situation;

- RSE payload cross-calibration activities support;

- estimation of RSE satellite orbit parameters influence on the final product;

- $\quad$ keeping the history of orbit maneuver activity of SO;

- chronological research, including archive images of a given territory search;

- planning the placement of new objects of ground-based space infrastructure, including tracking stations of all types, optical and radio telescopes, launching sites, remote sensing data receiving stations, etc.;

- modeling of space objects tracking conditions using shape and size data;

- coordinate and time referencing of cataloged SO based on their images against the background of the star-field;

- $\quad$ surveillance facilities design;

- detecting the intersection of SO orbits and the collision risk estimation;

- updates and notifications transfer to other organizations and departments of the space industry;

- possibility assessment of the satellites constellation deployment in given orbits; 
- providing measures of space navigation and laser ranging, etc.

The research was carried out within the state assignment 0708-2020-0001 of the Ministry of Science and Higher Education of the Russian Federation.

\section{References}

1. L.S. Novikov, Fundamentals of near-Earth space's ecology, 84 (Universitetskaya kniga, 2006)

2. I.V. Barmin, V.P. Kulagin, V.P. Savinykh, V.Y. Tsvetkov, Solar System Research, 48, 531 (2014)

3. K.I. Kukk, Connect WIT, 7-8, 20 (2015)

4. I.V. Barmin, D.W. Dunham, V.P. Kulagin, V.P. Savinykh, V.Y. Tsvetkov, Solar System Research, 48, 593 (2014)

5. V.P. Savinykh, Perspectives of science and education, 2, 9 (2014)

6. V.P. Savinykh, V.P. Vasiliev, Yu.S. Kapranov, I.I. Krasnorylov, G.E. Kufahl, S.V. Perminov, V.V. Shevchenko, Izvestia vuzov «Geodesy and Aerophotosurveying», 2, 3 (2013)

7. D.J. Kessler, B.G. Cour-Palais, J. Geophys. Res., 83, 2637 (1978)

8. H. Klinkrad, Advances in Space Research, 11, 43 (1991)

9. P.Yu. Orlov, A.E. Lugovskoi, Izvestia vuzov «Geodesy and Aerophotosurveying». (to be published)

10. URL: http://orbitaldebris.jsc.nasa.gov/index.html

11. URL: https://comspoc.com/

12. T.S. Kelso, Advances in the Astronautical Sciences, 135, 1099 (2009)

13. T. Wang, Science \& Global Security, 18, 87 (2010)

14. T.S. Kelso, Proceedings of the $8^{\text {th }}$ Advanced Maui Optical and Space Surveillance Technologies Conference, 1, 321 (2007)

15. Y. Jiang, Heliyon, 6, e04692 (2020)

16. URL: https://discosweb.esoc.esa.int/

17. T. Wang, Science \& Global Security, 24, 22 (2016)

18. P.Yu. Orlov, Razrabotka i issledovanie metodiki geoinformatsionnogo modelirovaniya trekhmernykh dinamicheskikh stsen okolozemnogo kosmicheskogo prostranstva. Development and research of the geo-information modeling technique of near-Earth space' three-dimensional dynamic scenes. PhD. Moscow: MIIGAiK, 2019: $190 \mathrm{p}$.

19. I.G. Zhurkin, S.S. Gruzdev, Izvestia vuzov «Geodesy and Aerophotosurveying», 4, 45 (2012)

20. S.S. Gruzdev, A.V. Pankin, Ecology, economics, informatics. Geoinformational technologies and satellite monitoring. Conference proceedings, 2, 267 (2013)

21. S.S. Gruzdev, Ecology, economics, informatics. Geoinformational technologies and satellite monitoring. Conference proceedings, 2, 321 (2013)

22. URL: https://www.agi.com/

23. P.Yu. Orlov, I.G. Zhurkin, Scientific Visualization, 10, 58 (2018)

24. D.E. Okhotsimskii, Yu.G. Sikharulidze, Fundamentals of space flight mechanics, 448 (Nauka, 1990)

25. URL: http://hyperwave.ulsu.ru 
26. URL: http://www.ucsusa.org/nuclear-weapons/space-weapons/satellite-database

27. I.G. Zhurkin, S.V. Shaytura, Geographic information systems, 272 (KUDITS-PRESS, 2009)

28. T. Wang, What's Up: A Visual Database of Satellites and Debris, 17 (Manual, version $1.1,2010)$

29. P.Yu. Orlov, M.A. Boyarchuk, I.G. Zhurkin, V.V. Nekrasov, Geodesy and Cartography, 12, 31 (2020)

30. D.A. Vallado, P. Crawford, R. Hujsak, T.S. Kelso, AIAA/AAS Astrodynamics Specialist Conference and Exhibit, AIAA 2006-6753 (2006)

31. T.S. Kelso, Satellite Times, 4, 52 (1998)

32. URL: http://www.celestrak.com/

33. URL: https://www.space-track.org/

34. D.A. Vallado, W.D. McClain, Fundamentals of astrodynamics and applications, 1106 (Microcosm Press, 2013)

35. URL: http://spacedata.vimpel.ru/

36. L. Zhu, Z. Wang, Z. Li, ISPRS Int. J. Geo-Inf., 7, 97 (2018)

37. L. Zhu, Z. Li, Z. Wang, ISPRS Int. J. Geo-Inf., 7, 102 (2018)

38. T.V. Bordovitsyna, A.G. Aleksandrova, I.N. Chuvashov, Vestnik Tomskogo gosudarstvennogo universiteta. Matematika i mekhanika, 4, 34 (2011)

39. V.I. Krylov, Coordinate-temporal transformations in geodesy, 90 (MIIGAiK, 2014)

40. V.I. Krylov, Space geodesy, 168 (MIIGAiK, 2002)

41. W. Ley, K. Wittmann, W. Hallmann, Handbook of space technology, 908 (Wiley, 2009)

42. M.A. Boyarchuk, Razrabotka i issledovanie metoda otobrazheniya vektornogo gravitatsionnogo polya Zemli dlya geoinformatsionnogo analiza. Development and research of the Earth's vector gravitational field displaying method for geoinformation analysis. PhD. Moscow: MIIGAiK, 2020: 128 p.

43. URL: https://puzzle-hexagon.glitch.me/

44. V.P. Vasilyev, V.D. Shargorodsky, Photonics, 6, 74 (2017)

45. V.P. Savinych, Russian Journal of Astrophysical Research. Series A, 5, 41 (2019) 